\title{
Solar Powered Fault Detection System for Railway Tracks
}

\author{
Alia Rifat, P. P. Pandao and B. Shoban Babu
}

\begin{abstract}
Indian Railways has one of the world's largest networks. Train movement is always reliant on railway rails alone. If one of these rails develops a crack, it becomes a huge issue. Many railway accidents occur as a result of the presence of a crack. The most difficult aspect of a railway analysis is detecting structural faults. If these flaws aren't addressed early on, they could lead to a series of derailments, resulting in a significant loss of life and property. The proposed railway track error detection system detects faulty railway tracks automatically and without the need for human intervention. This project intends to create a solar based autonomous railway track crack detection vehicle that uses a microcontroller and ultrasonic sensors to detect cracks along its journey. The ultrasonic sensor detects the fracture and objects, sending the information to the microcontroller, which quickly stops the train. Solar panels are utilized in this vehicle to absorb solar energy [1], which is then transformed into electrical energy, which is then used to charge a lead acid battery, which then provides the necessary power to a DC motor. This energy is subsequently sent to the DC motor, which drives the wheels. Sensors are utilized to detect cracks, and if a crack is detected, the system will halt and sound an alarm.
\end{abstract}

Keywords — faults, microcontroller, railway, track crack.

\section{INTRODUCTION}

Transportation is a critical need for action since it allows for the production and usage of things to occur in a variety of locations. Increasing the capacity and reliability of transportation has always been critical to economic growth. However, transportation infrastructure and efficiency have a big impact on the world and are a key source of energy, making transportation stability and security a significant concern. Trains are essential for transporting passengers and products from one location to another. More trade results from a better transportation system. It is critical to protect the safety of these diverse modes of transportation, particularly as railway networks play a significant role in India's economic and transportation infrastructure. The Indian Railway network is the world's largest transit network and the country's transportation infrastructure's backbone. The railway network makes up the majority of commercial transportation in India because it is the cheapest mode of transit before all other modes of transportation such

Submitted on January 10, 2022

Published on February 05, 2022.

Alia Rifat, Research associate, LeenaBOT Robotics LLC, USA.

(e-mail: jodhyy@gmail.com)

P. P. Pandao, LeenaBOT Robotics pvt Ltd, India.

(e-mail: p3pandao@gmail.com)

Dr. B. Shoban Babu, SV Engineering College, Tirupati, India.

(e-mail: bshobanbabu@gmail.com) as buses, aero planes, and so on. In recent years, India's fastgrowing economy has resulted in increased demand for transportation, resulting in a remarkable increase in traffic volume on the Indian Railway network. In India, we find that rail transportation plays a critical role in supplying the transportation infrastructure required to sustain and eradicate the fast-growing economy's ever-increasing needs. India now possesses the world's fourth largest railway network. However, we are still far from meeting the international level. Every element of our everyday life has been impacted by automation. In practically every industry, new development is being implemented to reduce human effort and save time [2]. The railway track crack is shown in Fig. 1.

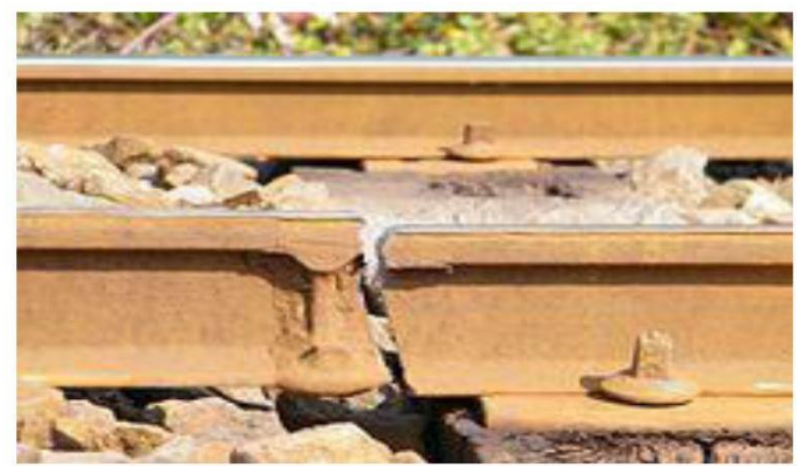

Fig. 1. Crack in a Railway Track.

The Internet of Things (IoT) is the expansion of Internet connectivity into physical devices and everyday items. These devices, which are equipped with a variety of gadgets and equipment (such as sensors, cameras, and motors), may communicate with one another through the Internet and be monitored and controlled remotely [3]. The Internet of Things (IoT) is connected to automation. In reality, one of the most important aspects of automation is the Internet of Things. The Internet of Things has taken on new significance as a result of the convergence of many advancements, ongoing research, AI, item sensors, and embedded frameworks. Embedded structures, remote sensor frameworks, control systems, motorization (monitoring home and building computerization), and other traditional sectors all contribute to connecting with the Internet of things. IoT innovations are increasingly identifying with the prospect of the "Smart home" in the buyer market, encompassing gadgets and apparatuses such as smart lighting, indoor regulators, home security and cameras, and other home machines [4]. It benefits at least one regular environment and can be controlled by biological systemrelated devices such as mobile phones and smart speakers. 


\section{LITERATURE REVIEW}

De Benedetti et al. [5] reported an algorithm for abnormality identification and predictive maintenance. This was determined by a comparison between measured and estimated AC power. The model was created to estimate power and was based on an artificial neural network that could forecast power output based on measured irradiance and temperature parameters. It was trained using data acquired from the PV system earlier. The technology was experimentally tested and evaluated with a $90 \%$ precision, according to the authors.

Rao et al. [6] implement a system for fault detection using neural networks, which is similar to the approach presented in this article However, in this case, they take into account the detection of eight different faults that are commonly thought to affect energy production efficiency. The authors demonstrate higher than 99 percent accuracy in this solution.

Pahwa et al. [7] compare the performance of Decision Tree, XGBoost, Random Forest, and Neural Networksbased classifiers for the automatic classification of defects in photovoltaic panels. The neural network classifier has the maximum accuracy of more than 99.5 percent, according to simulation findings (and, therefore, the lowest mean square error).

Firth et al. [8] created a simple characterization modelling PV systems' "normal operation," that is, when no problems occur. Environmental temperature, panel temperature, panel irradiance, and panel power output were all monitored in this study. Two tests were conducted at distinct locations, with data collected every 5 minutes for two years in the first and one year in the second. After that, a model was built in which the energy efficiency of the system was estimated with the goal of determining its link to power output and irradiance received by the system's area. After that, irradiance intervals were generated, and it was demonstrated that each interval had a normal distribution. In other words, there are 20 normal distributions for every 20 irradiance intervals. As a result, if the efficiency considerably deviates from the respective normal distribution's mean for a given irradiance, a system problem might be deduced.

Bonsignore et al. [9] introduced an evolutionary method based on fuzzy logic and neural networks. When real-time data and the potentiality of fuzzy systems are utilized to form links between input and output variables, this method is stated to have the advantage of learning via experience (neural networks, training, and testing phases), resulting in a very exact output. As a result, in this study, the Adaptive Neuro-Fuzzy Inference System (ANFIS) technique was chosen. Module temperature, global irradiance on the system's plane, open circuit voltage, short-circuit current, and voltage and current at maximum power were among the six characteristics examined. From this data, characteristic I-V curves based on irradiances and temperatures were constructed, enabling for the identification of probable defects using the aforementioned expected versus obtained curves. The downside in this scenario is that errors are not detected on-line; instead, a periodic system check is necessary. That is, the system must be turned off in order to be verified, because measuring open-circuit voltage and short-circuit current necessitates unplugging the system.

\section{PROBLEM STATEMENTS}

A broken rail is one of the most common causes of the world's most expensive and dangerous rail accidents. In terms of overall wrecks, more than one notable wreck occurs every three days, on average, during the course of ten years in the United States. The data on the frequency of broken rail crashes in different countries is insufficient for a proper assessment of the financial, social, and environmental consequences. When the track is free, the proposed framework is made to hurry back and forth down the track at unequal intervals. Furthermore, if it detects any splits on the track, it will transmit a warning signal to the specialist via a remote module. Splits are detected using infrared sensors, and a mistake flag is sent.

\section{Design of a RaIlWAy TRACK FaUlt Detection SYSTEM BASED ON SOLAR ENERGY}

The block diagram for the proposed work, "Solar based railway track fault detection system," is shown below. The Arduino Uno microcontroller, which serves as the system's brain, is used in this project. This microcontroller is in charge of the circuit's operation. This microcontroller interfaces with a variety of components in order to fulfil the system's specified functions [10]. The operation of the hardware components in this system necessitates a controlled power source. The rechargeable battery linked to the system provides this power. With the help of a solar panel, the battery will be charged using solar energy as shown in Fig. 2.



Fig. 2. Block diagram

We connected two ultrasonic sensors to the microcontroller in this system to measure distance and detect a crack in the railway line's track. The GSM module facilitates communication between the user and the car. The latitude and longitude coordinates are sent to the appropriate authority through SMS using the GSM module. A GPS receiver is also connected to the microcontroller in order to pinpoint the precise location of the railway track crack. The longitude and latitude parameter values will be sent to the controller through SMS using this GPS receiver. The robot moves forward with the help of two DC motors. The microcontroller is used to connect and operate these motors. Two channel relays are necessary for connecting between the microcontroller and the motors in order to control these motors using a microcontroller. 


\section{A. Working}

This technology detects cracks based on image processing, which is a unique approach to detecting railway track cracks [11]. To detect railway track cracks, many picture preparation procedures are used. Because the image is susceptible to noise. The system converts the image to grayscale and then filters it to remove noise. Noise reduction aids in the accurate detection of cracks. The brightness of the image is raised, and it is turned to a binary image. This enables the system to detect only cracks while also assisting in the removal of other unwanted things. After converting the image to a binary format, holes are filled using an image processing method, which helps to eliminate all smaller items that aren't needed for crack detection. The intensity value is used to detect cracks. The intensity value is used to determine accuracy. Large blobs are detected using the blob analysis method. The number of connected components in the system is used to detect cracks [12]. The system detects a crack based on the number of blobs involved and indicates whether or not a fracture exists. The system displays a rectangular rectangle around the blob using bounding box functionality. This technique is used to check railway tracks. Ultrasonic sensors are connected to the Arduino Uno microcontroller. When the sensor detects a track issue, it communicates the information to Arduino. The vehicle's journey is paused, and the GSM module transmits information about the malfunction to the control room or railway station. As a result, the control room is informed about the location of the crack. This will speed up the tracking process. The proposed system can detect deeper cracks with an accuracy of $80 \%$ and minor cracks with a $50 \%-60 \%$ accuracy rate.

\section{COMPONENT AND SPECIFICATIONS}

\section{A. IR Sensor}

A producer (emitter), identifier (detector), and supporting hardware make up an IR sensor. The circuit required to build an IR sensor is divided into two sections: the producer circuit, which includes an emitter circuit, and the collector circuit, which includes a collection circuit. The producer (emitter) was simply an infrared LED (Light Emitting Diode), and the finder was simply an infrared photodiode sensitive to IR light of a wavelength similar to that transmitted by the IR LED. The IR sensor is shown in Fig. 3.

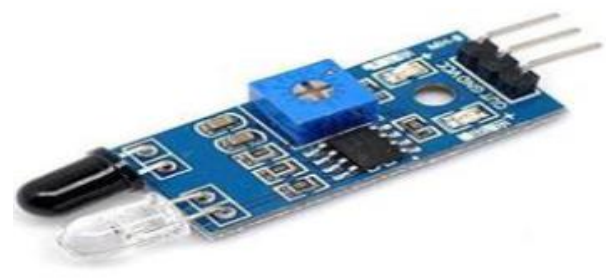

Fig. 3. IR Sensor.

\section{B. Ultrasonic Sensor}

VCC, lever, reverberation (echo), and earthing are the pins of an ultrasonic sensor with a four-pin configuration. By decoding the reflected sign, it is able to focus [13]. The sensor generates ultrasonic sound waves by converting electrical energy into sound, and then converts the sound waves into electrical vitality after receiving the reverberation signal.

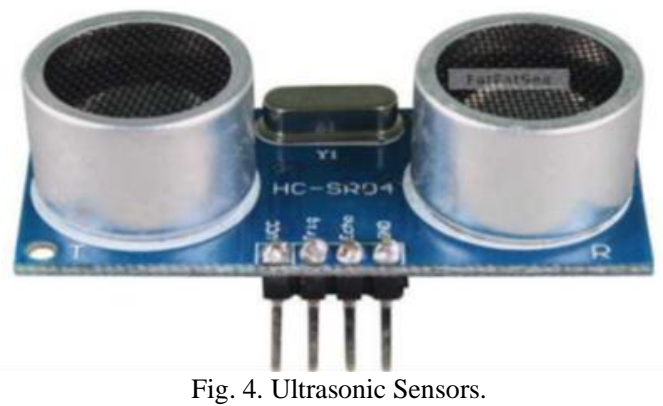

\section{Arduino UNO}

The Arduino UNO is a chipset that runs on the AT Mega328 microcontroller as shown in Fig 2. The Arduino UNO has 14 digital input and output pins, six of which can be utilised as Pulse Width Modulation outputs and the other six as analogue inputs. It has a resonant circuit with a frequency of sixteen megahertz (MHz), a USB connection, a power jack, a header, and a reset button. It also includes everything required to run the microcontroller. To start the chipset, we can connect it to a machine with a USB cable and power it with an adapter (DC current) or a battery.

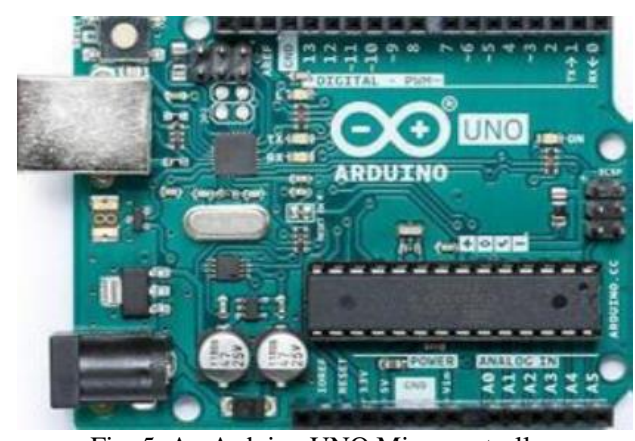

Fig. 5. An Arduino UNO Microcontroller.

\section{GSM Module}

A GSM Modem consists of a dedicated modem with a sequencer, USB Bluetooth connection, or mobile phones having GSM modem capacity or capabilities [14]. A GSM modem allows an application to send and receive messages over the modem interface, such as SMS. It is critical that a G SM modem assist a reached out AT Command set for sending/accepting SMS in order to do this task.

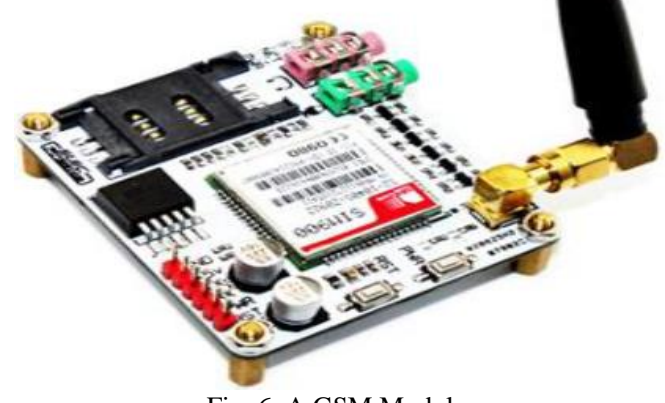

Fig. 6. A GSM Module. 


\section{E. GPRS Module}

The Global Positioning System (GPS) is a satellite route structure that is used to find/locate an item's ground position [15]. A GPS beneficiary accurately determines its function by timing the signal sent by GPS satellites orbiting far above the planet. A moving map show or scope (latitude) and longitude are then used to prove the position. GPS makes use of a lot of technical technology, yet the concept is simple. Every GPS satellite sends a signal to the GPS receiver. The satellites relay the exact time the signal's unit was sent [16]. The GPS can tell how far away it is from each satellite by subtracting the time the signal was transmitted from the time it was received.

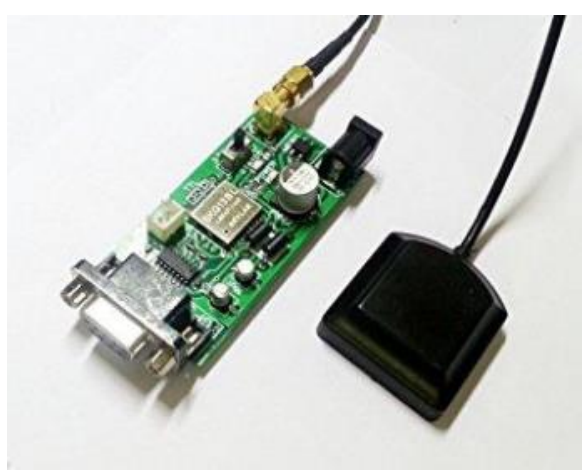

Fig. 7. A GPS Module.

\section{F. Motor Driver}

The integrated circuit L293D is a dual H-bridge motor driver (IC). Because they take a low-current control signal and convert it to a higher-current signal, motor drivers operate as current amplifiers.

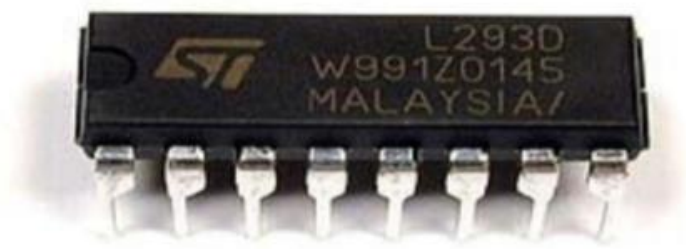

Fig. 8. Motor driver.

\section{G. Flow Chart}

The flowchart below (Fig. 8) depicts the steps involved in the operation of our model step by step.

\section{HARDWARE IMPLEMENTATION}

When the inspection robot is placed on the track, it uses IR sensors to detect any faults and sends a message to the android smartphone using the Bluetooth module. It also shows the message for a specific point on the track, such as left side crack or right-side crack [17]. It also keeps track of the track's GPS location as shown in Fig. 10 and 11.

\section{A. Case (I)}

When the track is fault-free, the Bluetooth module sends the message "TRACK IS GOOD" to the Android phone.

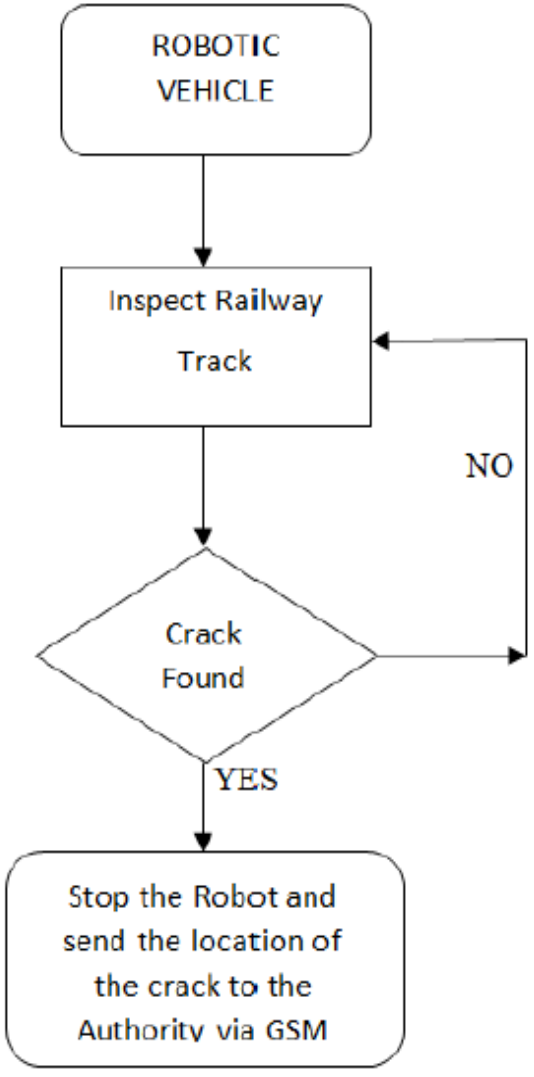

Fig. 9. Proposed Method Flow Chart

\section{B. Case (II)}

When a defect occurs on the track as depicted in, the notification "TRACK IS MISMATCH" appears on the Android phone, along with GPS tracking.

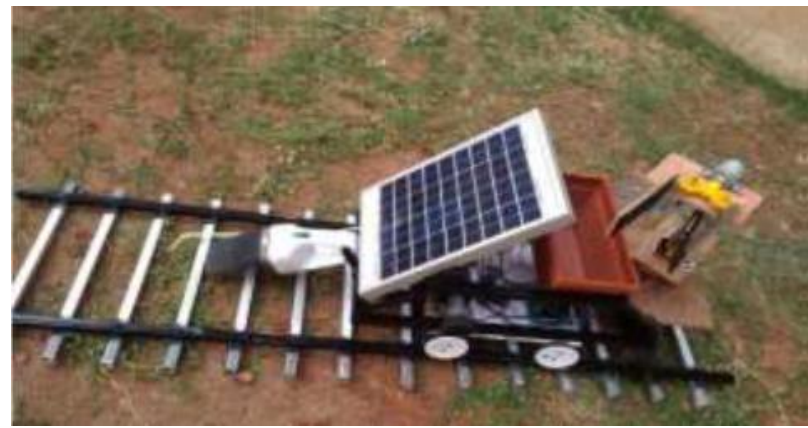

Fig. 10. Prototype

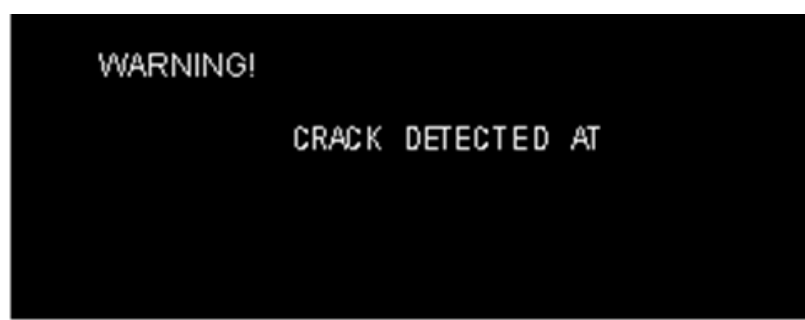

Fig .11. SMS

\section{CONCLUSION}

According to our proposed model, by designing this inspection system in real time, we will be able to quickly eliminate accidents caused by track side problems. The crack detection system is the most important part of the 
system [18]. The IOT module assists in notifying railway authorities of a track crack. As a result, this study proposes a crack detection and security system that is more reliable, less expensive, uses less power, takes less time to analyze, and requires fewer people. The precise location of the lives can be saved with this proposed approach. Along with fracture detection, the suggested system uses a suction mechanism, a robotic arm, and a watering mechanism to clean the railway track. The suction mechanism aids in the removal of small particles from the track. The robotic arm is used to pick up the crushed water bottles that have strewn across the track [19]. The complete system is monitored by using the mobile application to view video captured from the IP camera.

\section{A. Future Scope}

CCTV systems with IP-based cameras may be utilized in the future to monitor visual videos taken from the track. Passengers and railways will both benefit from the increased protection. by Using IR Sensors and Bluetooth Technology. Asian Journal of Applied Science and Technology (AJAST), 2017;1(6):82-84.

[16] R. Nagarajan, R. Yuvaraj, V. Hemalatha, S. Logapriya, A. Mekala and S. Priyanga. Implementation of PV - Based Boost Converter Using PI Controller with PSO Algorithm. International Journal of Engineering and Computer Science (IJECS); 2017, 6(3): 2047920484

[17] M. Kavitha and V. Sivachidambaranathan. Power factor correction in fuzzy based brushless DC motor fed by bridgeless buck boost converter. In International Conference on Computation of Power, Energy Information and Communication (ICCPEIC), 2017, pp. 549553, IEEE.

[18] P. A. Anand, S. Muley, B. P. Siddhant. Railway track crack detection based on GSM technique. International Research Journal of Engineering and Technology (IRJET), 2017;4(1):1252-1254.

[19] P. Kanade, P. Alva, S. Ghatwal, S. Kanade, Automated Robot ARM using Ultrasonic Sensor in Assembly Line. International Research Journal of Engineering and Technology (IRJET), 2020;7(12):615620

\section{REFERENCES}

[1] C. Ranhotigamage and S. C. Mukhopadhyay. Field trials and performance monitoring of distributed solar panels using a lowcost wireless sensors network for domestic applications. IEEE Sensors Journal, 2011; 11(10):2583-2590.

[2] U. B. Parikh, B.Das, and R. P.Maheshwari. Combined wavelet- SVM technique for fault zone detection in a series compensated transmission line. IEEE Transactions on PowerDelivery, 2008; 23(4); 1789-1794.

[3] Prakash Kanade, Fortune David, Sunay Kanade. Convolutional Neural Networks (CNN) based Eye-Gaze Tracking System using Machine Learning Algorithm. European Journal of Electrical Engineering and Computer Science, 2021; 5(2); 36-40.

[4] P Kanade, S Kanade. Raspberry Pi Project-Voice Controlled Robotic Assistant for Senior Citizens. International Research Journal of Engineering and Technology (IRJET), 2020; 7 (10): 1044-1049.

[5] M. Benedetti, F. Leonardi, Messina, C. Santoro \& A. Vasilakos. Anomaly detection and predictive maintenance for photovoltaic systems. Neurocomputing, 2018; 3(10): 59-68.

[6] S. Rao, A. Spanias \& C. Tepedelenlioglu. Solar array fault detection using neural networks. IEEE International Conference on Industrial Cyber Physical Systems (ICPS), 2019; 196-200, Taipei, Taiwan.

[7] K. Pahwa Sharma, M. Saggu \& A. K. Mandpura. Performance evaluation of machine learning techniques for fault detection and classification in PV array systems. 7th International Conference on Signal Processing and Integrated Networks (SPIN), 2020; 791-796. IEEE, Noida, India.

[8] S. K. Firth, K. J. Lomas \& S. J. Rees. A simple model of PV system performance and its use in fault detection. Solar Energy, 2010; 84(4): 624-635.

[9] L. Bonsignore, M. Davarifar, A. Rabhi, G. M. Tina \& A. Elhajjaji. Neuro-Fuzzy fault detection method for photovoltaic systems. Energy Procedia, 2014;62(5):431-441.

[10] B. R. BalaJaswanth and C. Muneendra Rao. Crack Sensing Scheme in Rail Tracking System. International Journal of Engineering Research and Applications, 2014;4(1):13-18.

[11] U. Mishra, V. Gupta, S. M. Ahzam and S. M. Tripathi. Google Map Based Railway Track Fault Detection over the Internet. International Journal of Applied Engineering Research, 2019;14(2):20-23.

[12] K Prakash, A Prajna. Raspberry PI Project - Ultrasonic Distance Sensor in Civil Engineering. International Journal in IT \& Engineering (IJITE), 2020;8(10):1-6.

[13] P Kanade, Jai Prakash Prasad, S Kanade. IOT based Smart Healthcare Wheelchair for Independent Elderly. European Journal of Electrical Engineering and Computer Science, 2021;5 (5): 4-9.

[14] P Kanade, P Alva, Jai Prakash Prasad, S Kanade, Smart Garbage Monitoring System using Internet of Things (IoT), 5th International Conference on Computing Methodologies and Communication (ICCMC), 2021:330-335, IEEE.

[15] B. Siva Rama Krishna, D. V. S Seshendra, G. Govinda Raja, T. Sudharshan, and K. Srikanth. Railway Track Fault Detection System 